Journal of Bionic Engineering 4 (2007) 53-62

\title{
Multisensor Data Fusion for High Quality Data Analysis and Processing in Measurement and Instrumentation
}

\author{
Yan-bo Huang ${ }^{1}$, Yu-bin Lan ${ }^{1}$, W. C. Hoffmann ${ }^{1}$, R. E. Lacey ${ }^{2}$ \\ 1. USDA ARS Agricultural Engineer, Areawide Pest Management Research Unit, Texas 77845, USA \\ 2. Biological and Agricultural Engineering Department, Texas A\&M University, Texas 77843-2117, USA
}

\begin{abstract}
Multisensor data fusion (MDF) is an emerging technology to fuse data from multiple sensors in order to make a more accurate estimation of the environment through measurement and detection. Applications of MDF cross a wide spectrum in military and civilian areas. With the rapid evolution of computers and the proliferation of micro-mechanical/electrical systems sensors, the utilization of MDF is being popularized in research and applications. This paper focuses on application of MDF for high quality data analysis and processing in measurement and instrumentation. A practical, general data fusion scheme was established on the basis of feature extraction and merge of data from multiple sensors. This scheme integrates artificial neural networks for high performance pattern recognition. A number of successful applications in areas of NDI (Non-Destructive Inspection) corrosion detection, food quality and safety characterization, and precision agriculture are described and discussed in order to motivate new applications in these or other areas. This paper gives an overall picture of using the MDF method to increase the accuracy of data analysis and processing in measurement and instrumentation in different areas of applications.

Keywords: multisensor data fusion, artificial neural networks, NDI, food quality and safety characterization, precision agriculture

Copyright $@$ 2007, Jilin University. Published by Science Press and Elsevier Limited. All rights reserved.
\end{abstract}

\section{Introduction}

Multisensor data fusion (MDF) is an emerging technology to fuse data from multiple sensors in order to make a more accurate estimation of the environment through measurement and detection. Applications of MDF cross a wide spectrum, including the areas in military services such as automatic target detection and tracking, battlefield surveillance, etc. and the areas in civilian applications such as environment surveillance and monitoring, monitoring of complex machinery, medical diagnosis, smart building, food quality characterization and even precision agriculture. Techniques for data fusion are integrated from a wide variety of disciplines, including signal processing, pattern recognition, and statistical estimation, artificial intelligence, and control theory. The rapid evolution of computers, proliferation of micro-mechanical/electrical systems sensors, and the maturation of data fusion technology provide a basis for utilization of data fusion in everyday applications $^{[1]}$.

This paper presents a number of applications of MDF for high quality data analysis and processing in measurement and instrumentation. The areas of the application have yet relatively few articles in engineering literature. The data fusion models of these applications are developed based on a general, practical MDF scheme for each specific area. This paper gives an overall picture of using the MDF method to increase the accuracy of data analysis and processing in measurement and instrumentation in different areas of applications.

\section{Technical Background}

Multi-sensor data fusion provides an approach to improving of performance of single sensor. In general, 
the data of measurement from a single sensor is limited to achieve a high quality. If a number of sensors can be used to perform the same measurement and the data of measurements from these sensors can be combined some way, the resultant has a great potential to out perform over each single measurement with increased accuracy.

In general, as its name implies, MDF is a technique by which data from a number of sensors are combined through a centralized data processor to provide comprehensive and accurate information. This technology has a powerful potential to track changing conditions and anticipate impacts more consistently than a single data source could do traditionally even this single data source might be a highly reliable one. Thus, MDF makes it possible to create a synergistic process, in which, the consolidation of individual data creates a combined resource with a productive value greater than the sum of its parts ${ }^{[2]}$.

Although the concept is not new, MDF technology is still in its infancy. This technology has undergone rapid growth that started in the late 1980s and has continued to the present. The U.S. Department of Defense (DoD) conducted much of the early research on this technology and explored its usefulness in military surveillance and land-based battle management systems. The application of data fusion technology to commercial endeavours (e.g.. robotics and general image processing) and non-military government projects (e.g., weather surveillance and NASA missions) is also growing rapidly. In its current state, the technology can combine sensor data of many types, including radar, infrared, sonar, and visual information.

In data analysis and processing of measurement and instrumentation, pattern recognition techniques are necessary. Pattern recognition is used to develop data fusion algorithms. Artificial neural networks, which have been developed based on studies about the mechanism of human brain, are the top option over other conventional statistical pattern recognition methods. Linn and Hall ${ }^{[3]}$ surveyed more than fifty data fusion systems. Only three of these systems used the neural network method. This low number may indicate an underestimation of the importance of neural networks in the field of data fusion.

Artificial neural networks have been widely used in solving complex problems, such as pattern recognition, fast information processing and adaptation. Artificial neural networks are structured based on studies about the mechanism and the structure of human brain. The architecture and implementation of a neural network models a simplified version of the structure and activities of the human brain. The vast processing power inherent in biological neural structures has inspired the study of the structure itself as a model for designing and organizing man-made computing structures ${ }^{[4-9]}$.

A MDF scheme integrated with neural network pattern recognition is a promising structure to achieve high quality data analysis and processing in measurement and instrumentation. This paper will emphasize the design and implementation of this structure.

\section{Method}

Three basic alternatives can be used for $\mathrm{MDF}^{[1]}$ :

(1) Direct fusion of sensor data;

(2) Representation of sensor data via feature vectors, with subsequent fusion of the feature vectors;

(3) Processing of each sensor to achieve high-level inferences or decision, which is subsequently combined.

Each of these approaches is motivated and influenced by some type of applications and utilizes different fusion methods. They can not be generalized to all data fusion methods. This paper will focus on the feature-based data fusion method.

With the measurement data from each sensor, the features can be quantitatively extracted from each sensor's output. The extracted feature vectors can be combined as the following matrix:

$$
\underline{\boldsymbol{F}}=\left[\underline{f}_{1} \underline{f}_{2} \ldots \underline{f}_{n}\right]^{\mathrm{T}} \quad(n \geq 2),
$$

where $f$ is the feature vector from the $i$ th sensor and $n$ is the number of sensors.

With each of the feature vectors as the input, a pattern recognition technique, typically a neural network is needed to differentiate the measurement data into appropriate classes. The output vectors of the $n$ neural networks are necessary to be merged for data fusion. 
Suppose $O_{1}^{i}, O_{2}^{i}, \cdots, O_{m}^{i}(i=1,2, \cdots, n)$ are the $m$ outputs of the neural network for the ith sensor. The merged output should be in the form:

$$
O_{j}^{M}=c_{1} O_{j}^{1}+c_{2} O_{j}^{2}+\cdots+c_{n} O_{j}^{n} \quad(j=1,2, \cdots, m)
$$

where $c_{1}, c_{2}, \ldots, c_{n}$ are the merging coefficients which can be determined by assigning values or by optimization.

Fig. 1 shows the diagram of the MDF scheme with neural networks. This scheme should be implemented as follows:

(1) Extract features from the measurement data of each of the $n$ sensors;

(2) Classify the feature vectors through a neural network classifier;

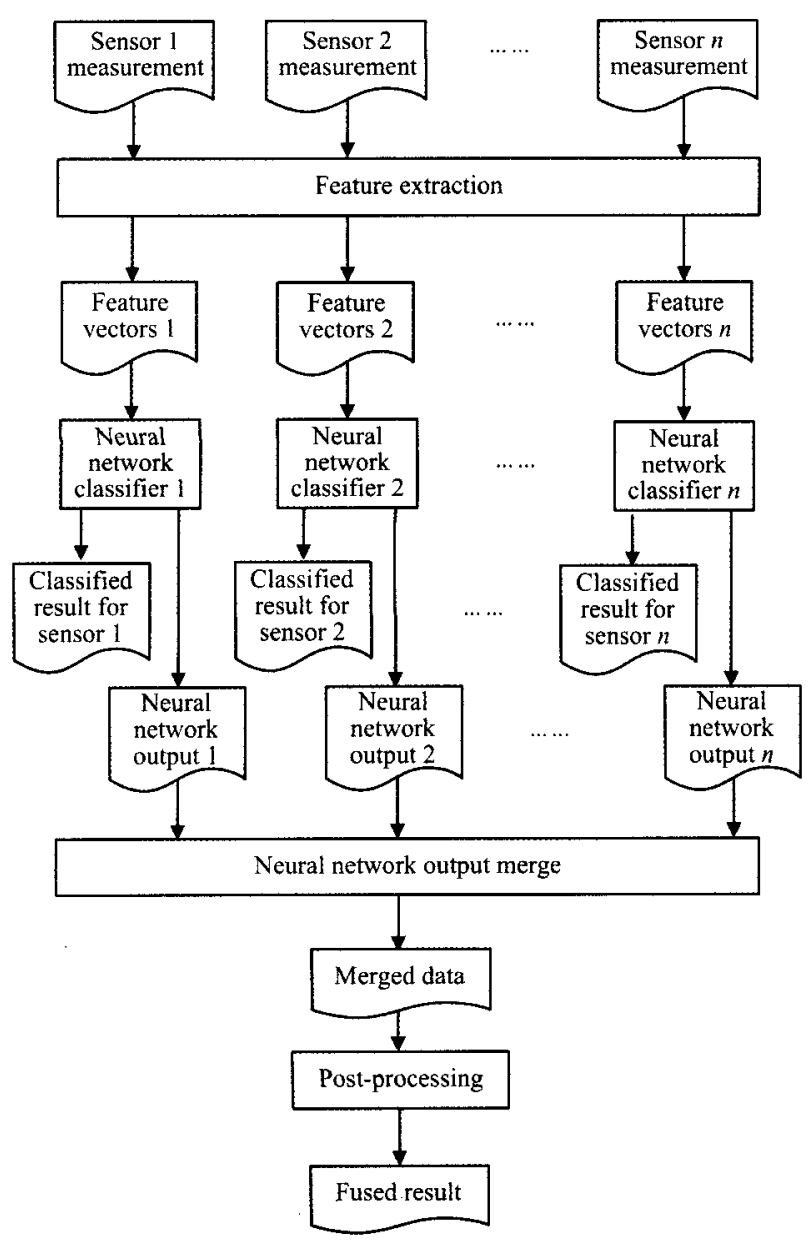

Fig. 1 Scheme diagram of multisensor data fusion with neural network classifiers.
(3) Merge the $m$ outputs of the neural network classifiers for all the $n$ sensors;

(4) Post process to produce a fused classification result.

\section{Applications}

We have developed and are going to develop a number of applications of MDF for high quality data analysis and processing in measurement and instrumentation. The areas of these applications have few articles in engineering literature. This section will present them as the following subsections.

\subsection{Corrosion NDI}

In 1977, Gros $^{[10]}$ wrote a book that is the first book to devote exclusively to multisensor integration and data fusion applied to NDT (Non-Destructive Testing) or NDI (Non-Destructive Inspection). This book provides detailed case studies and practical guidelines for readers wishing to explore NDT/NDI data fusion. Since then research and application of NDT/NDI data fusion have been conducted in various areas. However, a general data fusion system model capable of handling various applications is very difficult, if not impossible, to design ${ }^{[10]}$. Various data fusion models under the general concept are necessary for each specific area of research and application.

The NDI ultrasound and eddy current imaging methods were studied for corrosion detection for aging aircraft panels ${ }^{[11,12]}$. In order to increase the accuracy of the detection from the individual sensor, a NDI data fusion method was developed for the same application ${ }^{[13]}$.

We developed another NDI data fusion method integrated with artificial neural network classifiers. Ultrasonic and eddy current image data run through neural network classifiers to identify the corrosive spots on the same aircraft panel specimen. In order to evaluate the overall performance of the NDI imaging in corrosion detection, the fusion of the classified corrosion data from the two different imaging sensors is of the interest. The MDF is expected to have a complete picture of the method and more accurate corrosion detection. 
Fig. 2 shows the diagram of the NDI imaging data fusion scheme with neural network classifiers. This scheme can be implemented as follows:

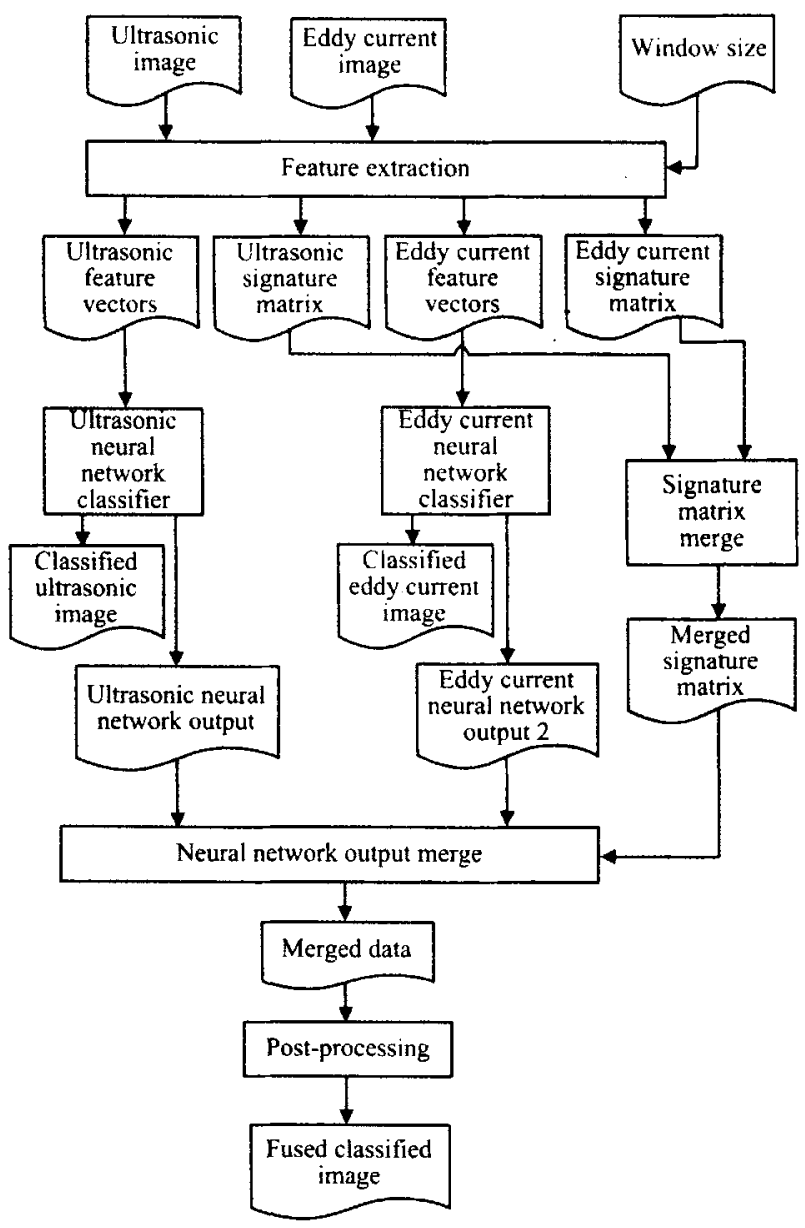

Fig. 2 Scheme diagram of corrosion NDI imaging data fusion with neural network classifiers.

(1) Extract statistical features from the ultrasonic and eddy current images with a window that goes over the images with a specified size: $2 \times 2,3 \times 3, \cdots$, or $12 \times 12$.

After feature extraction for each image, there are two sets of new data: feature vectors and signature matrix. The signature matrix is used to record what each pixel indicates in the image:

0 : indicates that this pixel in the image shows no corrosion;

1 : indicates that this pixel in the image may shows certain degree of corrosion but this pixel will not be included in neural network classification since the windowed feature extraction skipped it due to around it there are too many non-corrosive pixels;
2: indicates that this pixel in the image shows certain degree of corrosion and it will be included in neural network classification.

(2) Generate outputs of the neural network classfiers and produce classified ultrasonic and eddy current images.

(3) Merge the output vectors of the neural network classifiers for different sensors with the merged signature matrix.

The purpose of the signature matrix is to help find the relationship between the output sequences of neural network classifiers for different sensors. The merged signature matrix is formed as:

0 : when both are 0 at the same pixel in ultrasonic and eddy current signature matrices;

1: when either 0 or 1 at the same pixel in ultrasonic or eddy current signature matrices;

2: when at least a 2 at the same pixel in ultrasonic or eddy current signature matrices.

(4) Produce a fused classified image.

This scheme implements two merging functions: merge of feature signature matrices and merge of outputs and neural network classifiers for different sensors. The fused image can outperform over each individual image from the two different imaging sensors because the fused product integrates what the two different sensors captured.

The products of this scheme are: a classified ultrasonic image, a classified eddy current image, and a fused classified image. To evaluate the performance of the data fusion model, the three images were compared with a benchmark X-ray corrosion image of the same specimen (Fig. 3). Fig. 4 is an ultrasonic image of the corrosive aircraft panel specimen. Fig. 5 is the eddy current image of the specimen. Fig. 6 is the neural network classified ultrasonic image with a match rate of $50.61 \%$ with the X-ray data at $4 \times 4$ window size feature extraction. Fig. 7 is the neural network classified eddy current image with a match rate of $57.70 \%$ with the $\mathrm{X}$-ray data at $4 \times 4$ window size feature extraction. Fig. 8 is the fused classified image of Fig. 6 and Fig. 7. The match rate goes up to $65.49 \%$.

Table 1 summarizes the match rates of the data fusion of the neural network classifiers with the X-ray data 
at different window sizes. The results indicate that data fusion consistently enhances neural network corrosion detection with ultrasonic and eddy current image data individually.

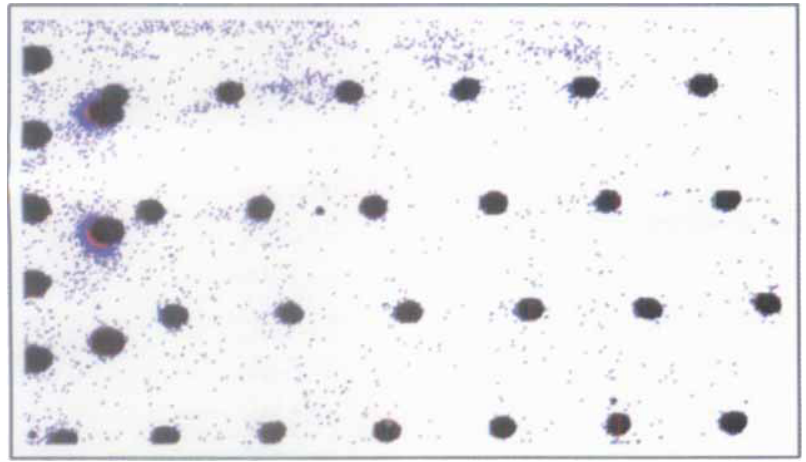

Fig. $3 \mathrm{X}$-ray image of a corrosive aircraft panel specimen on which the black pixels represent no corrosion, the blue pixels represent equal to or lower than $10 \%$ corrosion, and the red pixels represent higher than $10 \%$ corrosion.

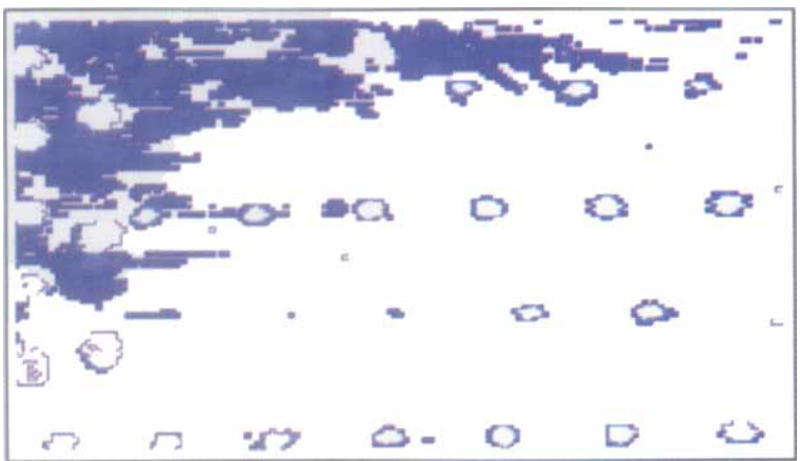

Fig. 4 Ultrasonic image of a corrosive aircraft panel specimen on which the blank pixels represent no corrosion and the color pixels represent corrosion.

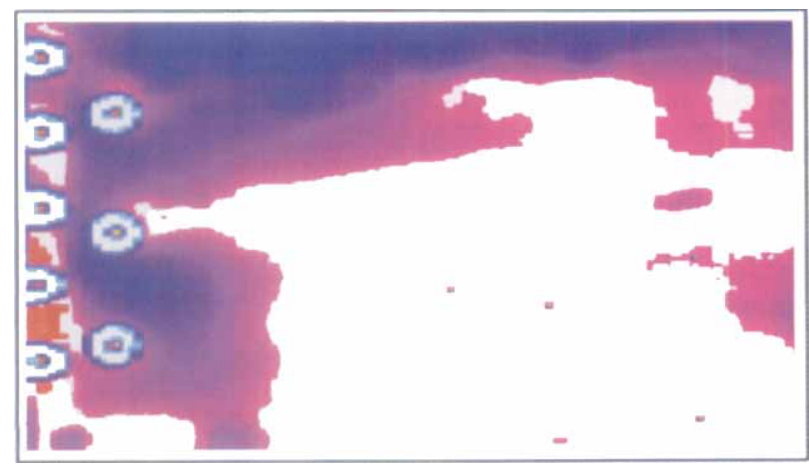

Fig. 5 Eddy current image of a corrosive aircraft panel specimen on which the blank pixels represent no corrosion and the colour pixels represent corrosion.

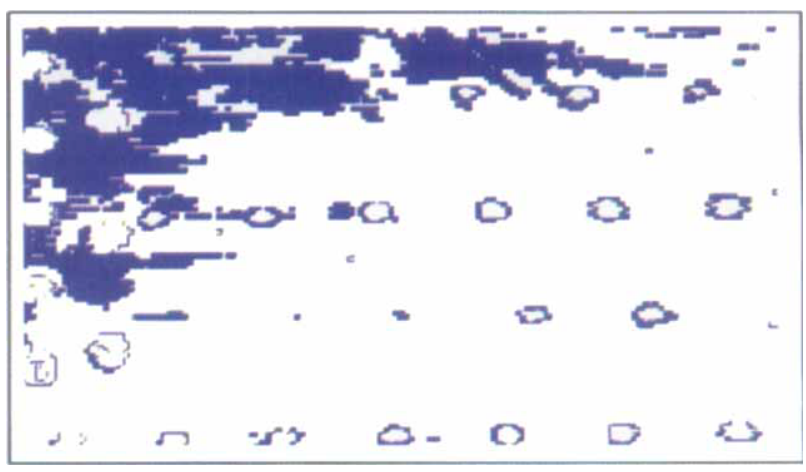

Fig. 6 Neural network classified ultrasonic image at $4 \times 4$ window size feature extraction on which the blank pixels represent no corrosion, the green pixels represent skipped during window shifting, the blue pixels represent low corrosion (equal to or lower than $10 \%$ corrosion), and the red pixels represent high corrosion (higher than $10 \%$ corrosion).

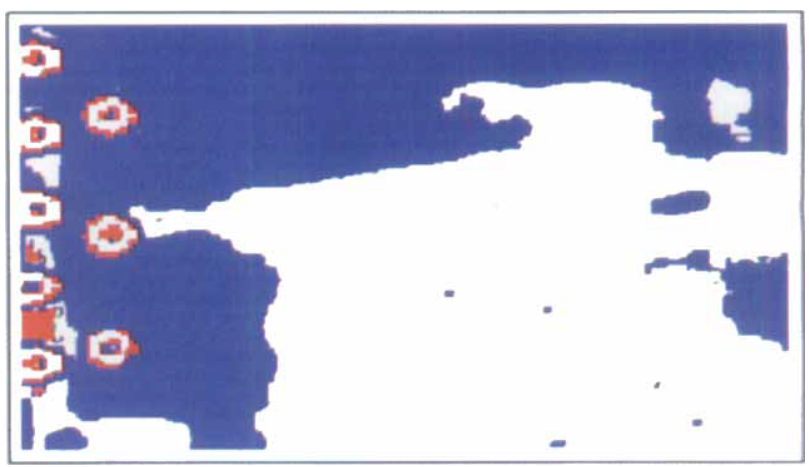

Fig. 7 Neural network classified eddy current image at $4 \times 4$ window size feature extraction on which the blank pixels represent no corrosion, the green pixels represent skipped during window shifting, the blue pixels represent low corrosion (equal to or lower than $10 \%$ corrosion), and the red pixels represent high corrosion (higher than $10 \%$ corrosion).

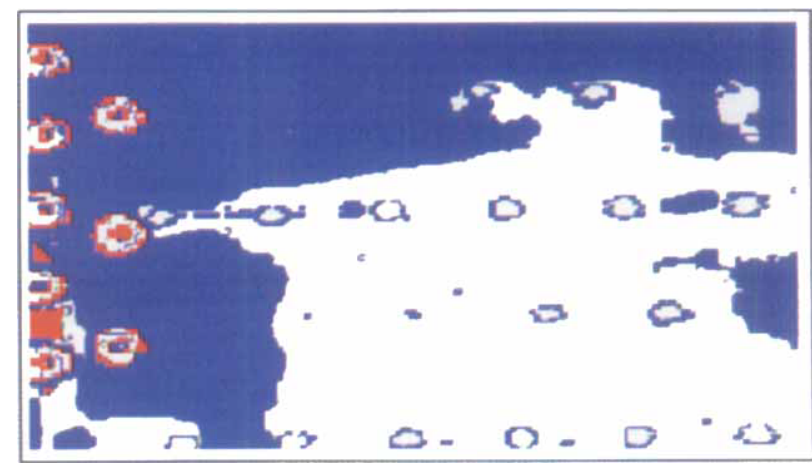

Fig. 8 Fused classified image from ultrasonic and eddy current neural network classified results on which the blank pixels represent no corrosion, the green pixels represent skipped during window shifting, the blue pixels represent low corrosion (equal to or lower than $10 \%$ corrosion), and the red pixels represent high corrosion (higher than 10\% corrosion). 
Journal of Bionic Engineering (2007) Vol.4 No.1

Table 1 Match rates of corrosion neural network classification with X-ray data with image feature extraction at different window sizes

\begin{tabular}{cccc}
\hline Window size & Ultrasonic & Eddy current & Data fusion \\
\hline $2 \times 2$ & $50.61 \%$ & $57.70 \%$ & $65.49 \%$ \\
$3 \times 3$ & $50.10 \%$ & $57.65 \%$ & $65.56 \%$ \\
$4 \times 4$ & $50.08 \%$ & $56.92 \%$ & $64.57 \%$ \\
$5 \times 5$ & $48.92 \%$ & $56.46 \%$ & $63.39 \%$ \\
$6 \times 6$ & $49.09 \%$ & $56.64 \%$ & $66.53 \%$ \\
$7 \times 7$ & $48.57 \%$ & $57.92 \%$ & $64.22 \%$ \\
$8 \times 8$ & $48.36 \%$ & $58.57 \%$ & $65.03 \%$ \\
$9 \times 9$ & $47.26 \%$ & $57.25 \%$ & $64.19 \%$ \\
$10 \times 10$ & $47.67 \%$ & $57.07 \%$ & $62.73 \%$ \\
$11 \times 11$ & $47.33 \%$ & $57.18 \%$ & $63.34 \%$ \\
$12 \times 12$ & $47.45 \%$ & $57.25 \%$ & $62.52 \%$ \\
\hline Average & $48.68 \%$ & $57.33 \%$ & $64.32 \%$ \\
\hline
\end{tabular}

\subsection{Food quality and safety characterization}

Food quality and safety are of primary concern in food industry and customer consuming. Sensor systems that are the mimic of human senses have been developed and applied for characterization of food quality ${ }^{[14]}$. Five basic human senses are: vision, hearing, smell, taste and touch. The senses are coordinated by the brain. In characterization of food quality and safety professional people sense the samples and provide data to differentiate "good" or "bad". However, the human sensory is usually subject to subjectivity, mental and physical inconsistencies, and lengthy time. Artificial senses such as machine vision, electronic ear, electronic nose, electronic tongue, artificial mouth, and even artificial head have been developed to mimic the human senses. These artificial senses are coordinated individually or collectively by a pattern recognition technique, typically also neural network classifiers. Such a structure has been used to formulate methods for rapid characterization of food quality ${ }^{[14]}$ and potentially for characterization of food safety.

With the concept of multi-sensor data fusion these sensor systems can work collectively. A simple fusion system can be formulated by fusing the measurements from a gas sensor and a taste sensor:

$$
\underline{F}=\left[\underline{f}_{\text {gas }} \underline{f}_{\text {taste }}\right]^{\mathrm{T}} .
$$

An artificial mouth ${ }^{[15,16]}$ can be formulated by fusing the measurements from an acoustic sensor, a gas sensor and a force sensor:

$$
\underline{F}=\left[\underline{f}_{\text {acoustic }} \underline{f}_{\text {gas }} \underline{f}_{\text {force }}\right]^{\mathrm{T}} .
$$

This artificial mouth was built by combining information corresponding to three senses "auditory" by a microphone, "tactile" by a force sensor and "olfaction" by a gas sensor array.

A piston in a chamber containing a crisp food sample was moved at a constant speed by the action of a stepping motor connected to a lever. The force applied to the piston was recorded by a force sensor, and a microphone was placed at the bottom of the chamber. Gas samples from the chamber were led to a sensor array consisting of ten MOSFET gas sensors, and four semi-conducting metal oxide type sensors.

The chamber system was exemplified by estimating aging and classifying different potato chips by collecting, analyzing and fusing crunching, hearing and smelling information. The research results indicate that individual information for tactile, smell and sound is not sufficient to follow the aging process of the chips. But when the individual information is merged, the aging process can be clearly followed by the fused information.

Artificial neural networks were able to predict the estimated aging process. Nine contributing features, 
three chosen from each of sensing source: aroma, sound, and tactile by principle component analysis, were fed into the network as the input. One output was the aging time. Seven nodes in the hidden layer were determined.

An artificial head ${ }^{[16,17]}$ is an electronic sensor head. It is a mimic of all five human senses to make a complete sensory evaluation of foods. The investigated food sample was entering an artificial mouth for detection of chewing resistance, measuring liberated aroma and recording to the chewing sound. A video camera was used for identifying colour, shape and other properties of the sample. Finally, the crushed sample was mixed with a saline solution, and an electrochemical multi-electrode arrangement was used to analyze the mixture.

An artificial head fuses the information completely from all five artificial sensor systems: vision, acoustic, gas, taste, and force. Features can be extracted from the information of each sensor system and combined together as the input to pattern recognition:

$$
\underline{\boldsymbol{F}}=\left[\underline{f}_{\text {vision }} \underline{f}_{\text {acoustic }} \underline{f}_{\text {gas }} \underline{f}_{\text {laste }} \underline{f}_{\text {force }}\right]^{\mathrm{T}} .
$$

Again artificial neural networks can be used to classify foods and to estimate quality parameters although a fuzzy classifier was suggested in the description.

This artificial head has been used for quality estimation of crispy products such as crisp bread and chips. The vision system alone could predict the freshness, spots and spiciness, that the olfactory is analogue to the amount of spiciness, and the auditory and touch are analogues to the freshness. Thus the freshness of the chips could be determined by change in colour and by change in texture. Also, the spiciness of the chip could be determined by the smell and by the number and colour of the spices as seen by the camera. So, if all senses were fused together, all quality parameters could be correctly predicted.

Fig. 9 gives a general picture of MDF for characterization of food quality and safety. In practice it has to decide which fusion system, artificial head, artificial mouth, or simple fusion, are needed based on information availability and research purposes.

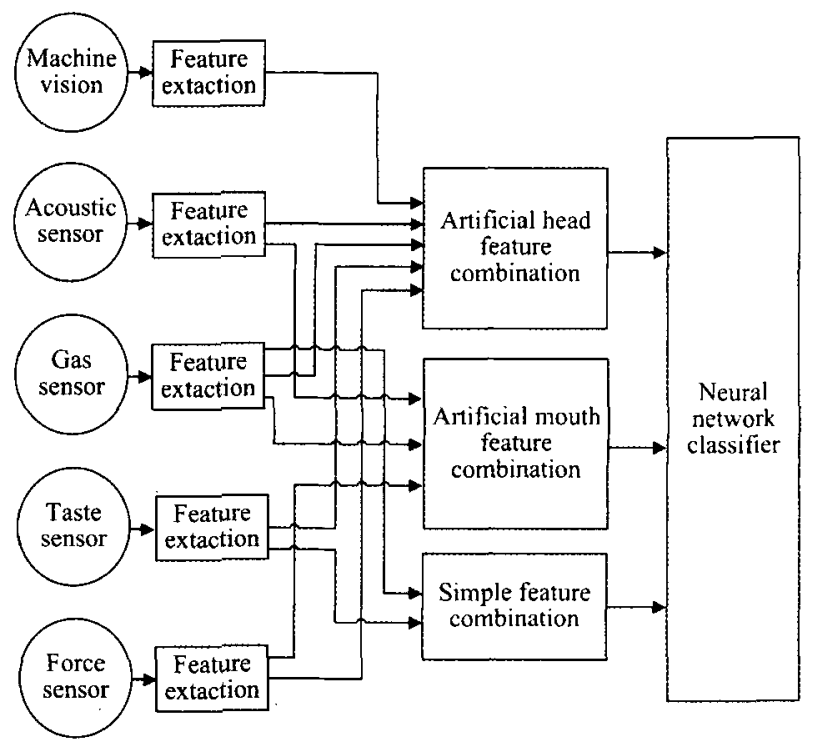

Fig. 9 Multisensor data fusion for characterization of food quality and safety.

\subsection{Precision agriculture}

Precision agriculture integrates a suite of technologies, especially GPS (Global Position System), GIS (Geographic Information System), and remote sensing. This integration retains the benefits of large-scale mechanization, which is essential to large fields, but recognizes local variation. By using satellite data to determine soil conditions and plant development, these technologies can lower the production cost by fine-tuning seeding, fertilizer, chemical and water use, and potentially increase production.

In precision agriculture, a large amount of data analysis and processing work is necessary for measurement and instrumentation. In order to increase the accuracy of the data analysis and the quality of the data processing, the method of MDF is more and more used in this area.

Researchers in Illinois Laboratory for Agricultural Remote Sensing have investigated the feasibility of using MDF to develop dependable crop system monitoring tools ${ }^{[18]}$. They used the MDF technology to increase field location sensor accuracy. They introduced a GPS-encoder hybrid positioning system. This hybrid system was composed of a differential GPS receiver and two optical encoders on the vehicle shafts. Integrating 
the GPS signal with the optical encoders output by curve matching can significantly improve the positioning resolution in both global range and single-point. Since the matched curve is based on the directional information from GPS and the curve shape from the optical encoder, the resulting resolution is much higher. The system retains the advantages of GPS and optical encoders, such as low cost, high-speed response and high resolution. To evaluate and validate this system, they have further developed a unique machine vision-based true path generator. The experiment results revealed that with the new hybrid system, the positioning accuracy of a ground truth machine has been significantly improved. The sensing system can meet the real-time spatial high resolution positioning demands in field operations with high-speed dynamic response.

A study was conducted to use MDF for measurements of the horizontal sprayer boom movements ${ }^{[19]}$. This study investigated the horizontal movements of trailed sprayer booms with the aim of distinguishing their yaw and jolt motions as well as their deformations. Two vehicles were equipped with a radar speed sensor and a three-axis dynamic measurement unit, while each boom was instrumented with ultrasonic sensors and accelerometers. Sensor fusion was used to extract yaw, jolt and deformation speeds by assuming that the motion of any point of the boom is equal to the superimposition of complementary high and low-frequency motions, delimited by a cut-off frequency, estimated on the basis of laboratory trials.

A wireless data fusion system was developed for agricultural vehicle positioning ${ }^{[20]}$. This wireless data fusion system was designed to automatically collect and process operational data from agricultural machinery in order to provide real-time support for precision farming tasks. The system provides transparent data management and decision support functions to crop producers by wirelessly integrating a machinery-based sensing unit and an office-based data processing unit. The machinery-based unit collects the machine position and attitude data, the office-based unit performs data fusion to accurately estimate the machine position, and the wireless data-link transmits the data between the machinery- and the office-based units. Field test results verify that this newly developed wireless data fusion system is capable of handling field data in real-time to support data-intensive precision farming operations.

A study of sensor fusion with a machine vision, an RTK-GPS, and a geometric direction sensor (GDS) was conducted to develop the guidance system for agriculture vehicle navigation ${ }^{[21]}$. This study adopted the EKF (Extened Kalman Filter) and a statistical method based on two-dimensional PDF (Probability Density Function). The developed navigation planner operated satisfactorily with an $8.4 \mathrm{~cm}$ average later error of vehicle guidance based on the data fusion of the RTK-GPS and the GDS.

A low-cost navigation system of autonomous agricultural vehicles was developed by sensor data fusion of a Garmin GPS and a set of solid inertial sensors ${ }^{[22,23]}$. Based on a positon-velocity-attitude (PVA) model of the vehicle, a PVA model-based Kalman filter was designed to integrate data from all sensors to provide accurate and robust vehicle positioning data. The accuracy of this developed system can meet most tasks in precision farming applications.

Sensor data fusion of a differential GPS receiver and a machine vision was developed in a fuzzy logic model for autonomous navigation of agricultural vehicles $^{[24]}$. The purpose of the development is to correct GPS tracking errors. The fusion philosophy is based on estimating the quality of each sensor output and obtaining the positioning corrections according to a quality-based weight. Filed experiments demonstrated the adequacy of using a local positioning sensor, the machine vision, to correct a GPS receiver's error through sensor fusion.

In the development of aerial precision sprayer for site specific pest management much research has been done with ground-based sprayers to reduce pesticide use and production costs, and to protect the environment. Technologies for aerial applications are becoming more available. One new approach for aerial application is variable rate technology (VRT), which consists of several precision agricultural technologies for applying a desired rate of crop production materials at a specific location. There is very limited application for commercial VRT devices due to their high cost and operational 
difficulty. An economic and user-friendly system is needed that could process spatially distributed information, and apply only the necessary amount of pesticides to the infested area, and much more efficient and minimize environmental damage. A key step in the system development is to develop prescription maps for aerial application using a remote sensing system and GPS/GIS technology. This makes it possible to perform multisensor, multi-spectral, multitemporal and even multi-resolution data fusion utilizing GIS technique to produce high quality prescription maps. The data fusion will be based on new methods for the fusion of heterogeneous data: numerical or measurable (radiometric, multi-spectral, and spatial information) and symbolic (thematic, human interpretation, and ground truth) data. The MDF scheme needs to be fully integrated into the system through GIS.

\section{Conclusions}

The method of MDF has been successfully applied in solving various problems in science and engineering. It is very promising for high quality data analysis and processing in measurement and instrumentation in various areas, including NDI, food quality and safety characterization, and precision agriculture. With more study and research, MDF will be used to solve problems in new areas, such as in the development of aerial precision sprayer for site specific pest management, in order to increase the measuring accuracy only with a single sensor.

A general MDF system model capable of handling various applications is very difficult. For a specific area of research and application, a specific MDF scheme under the general concept must be built. In different MDF schemes, artificial neural networks have been successfully applied for high performance pattern recognition in MDF even with an underestimation of the importance. They will be integrated into more and more schemes for high quality MDF.

To design a data fusion scheme, an important issue is to decide the level of fusion. Low level fusion directly adds up the data from different sensors. High level fusion is to analyze the features from each individual sensor system first and then to associate these features to produce a fused result. To determine the level of fusion for different problems is still an issue to study.

\section{References}

[1] Hall D L, Llinas J. Handbook of Multisensor Data Fusion, CRC Press LLC, USA, 2001.

[2] Hackett J K, Shah M. Multi-sensor fusion: A perspective. Proceedings 1990 IEEE International Conference on Robotics and Automation, 1990, 2, 1324-1330.

[3] Linn R J, Hall D L. A survey of multi-sensor data fusion systems. Proceedings of the SPIE - The International Society for Optical Engineering, SPIE, 1991, 1470, 13-29.

[4] McCulloch W S, Pitts W. A logical calculus of the ideas immanent in nervous activity. Bulletin of Mathematical Biophysics, 1943, 9, 127-147.

[5] Hebb D O. The Organization of Behavior, John Wiley \& Sons, New York, 1949.

[6] Rosenblatt F. The perceptron: A probabilistic model for information storage and organization in the brain. Psychological Review, 1958, 65, 386-408.

[7] Minsky M L, Papert S A. Perceptions, MIT Press, Cambridge, USA, 1969.

[8] Grossberg S. Adaptive pattern classification and universal recording: I. Parallel development and coding of neural feature detectors. Biological Cybernetics, 1976, 23, 121-134.

[9] Hopfield J J. Neural networks and physical systems with emergent collective computational abilities. Proceedings of the National Academy of Sciences, 1982, 79, 2554-2558.

[10] Gros X E. NDT Data Fusion, Arnold, London, 1997.

[11] Rebbapragada S, Palakal M J, Pidaparti R M, Jones C R. Corrosion detection and quantification using image processing for aging aircraft panels. The Third Joint FAADDoD/ NASA Conference on Aging Aircrafts, Albuquerque, NM, USA, 1999.

[12] Palakal M J, Pidaparti R M, Rebbapragada S, Jones C R. Intelligent computational methods for corrosion damage assessment. AIAA Journal, 2001, 39, 1936-1943.

[13] Forsyth D S, Liu Z, Komorowski J P, Peeler D. An application of NDI data fusion to aging aircraft structures. The 6th Joint FAA/DoD/NASA Conference on Aging Aircraft, San Francisco, USA, 2002.

[14] Huang Y, Lan Y, Lcaey R E. Artificial senses for characterization of food quality. Journal of Bionics Engineering, 2004, 
1, 159-173.

[15] Wide P, Winquist F, Lauber A. The perceiving sensory estimated in an artificial human estimation based sensor system. Proceeding of the IEEE Instrumentation and Measurement Technology Conference, Ottawa, Canada, 1997.

[16] Wide P, Winquist F, Kalaykov I. The artificial sensor head: A new approach in assessment of human based quality. Proceeding of the Second International Conference on Information Fusion, Sunnyvale, CA, USA, 1999, 2, 1144-1149.

[17] Huang Y, Lan Y, Lacey R E. Development of artificial head for characterization of food safety. ASAE Annual International Meeting, Tampa, FL, USA, 2005.

[18] Illinois Laboratory for Agricultural Remote Sensing, [20071-15], htp://www.age.uiuc.edu/remote-sensing/Sensing.html

[19] Ooms D, Lebeau F, Ruter R, Destain M F. Measurements of the horizontal sprayer boom movements by sensor data fusion. Computers and Electronics in Agriculture, 2002, 33, 139-162.

[20] Guo L S, Zhang Q. Wireless data fusion system for agricul- tural vehicle positioning. Biosystems Engineering, 2005, 91, 261-269.

[21] Noguchi N, Reid J F, Will J, Benson E R, Stombaugh T S. Vehicle automation system based on multi-sensor integration. ASAE Annual International Meeting, Orlando, FL, USA, 1998.

[22] Guo L S, Zhang Q. A low-cost navigation system for autonomous off-road vehicles. Proceedings of Automation Technology for Off-Road Equipment Conference, Kyoto, Japan, 2004, 107-119.

[23] Guo L S, Zhang Q, Feng L. A low-cost integrated positioning system of GPS and inertial sensors for autonomous agricultural vehicles. ASAE Annual International Meeting, Las Vegas, NV, USA, 2003.

[24] Rovira-Mas F, Han S, Wei J, Reid J F. Fuzzy logic model fro sensor fusion of machine vision and GPS in autonomous navigation. ASAE Annual Intemational Meeting. Tampa, FL, USA, 2005. 